\title{
Vertical or asymmetric nystagmus need not imply neurological disease
}

Fatima S Shawkat, Anthony Kriss, Dorothy Thompson, Isabelle Russell-Eggitt, David Taylor, Christopher Harris

\begin{abstract}
Aim-To indicate that congenital idiopathic nystagmus (CIN) and sensory defect nystagmus (SDN) can be vertical or asymmetric in some children.

Methods-Of 276 children presenting with nystagmus for electrophysiological testing, 14 were identified as having CIN or SDN, yet had a nystagmus which was either vertical $(n=11)$ or horizontal asymmetric $(n=3)$. Flash electroretinograms and flash and pattern visual evoked potentials (VEPs) were recorded in all patients. Eye movement assessment, including horizontal optokinetic nystagmus (OKN) testing, was carried out in $11 / 14$ patients. Results-Eight patients (seven with vertical, one with asymmetric horizontal nystagmus) had congenital cone dysfunction. One patient with vertical and another with asymmetric nystagmus had cone-rod dystrophy. One patient with vertical upbeat had congenital stationary night blindness. Two patients (one downbeat, one upbeat nystagmus) had normal electrophysiological, clinical, and brain magnetic resonance imaging findings and were classified as having CIN. One patient with asymmetric nystagmus showed electrophysiological and clinical findings associated with albinism. Horizontal $\mathrm{OKN}$ was present in $80 \%$ of patients tested, including the three cases with horizontal asymmetric nystagmus. This is atypical in both CIN and SDN, where the OKN is usually absent.

Conclusions-Vertical and asymmetric nystagmus are most commonly associated with serious intracranial pathology and its presence is an indication for neuroimaging studies. However, such nystagmus can occur in children with retinal disease, albinism, and in cases with CIN. These findings stress the importance of noninvasive VEP/ERG testing in all cases of typical and also atypical nystagmus.

(Br F Ophthalmol 2000;84:175-180)
\end{abstract}

Early onset nystagmus, which usually presents within the first 6 months of life, can be divided into three categories: congenital idiopathic nystagmus (CIN), sensory defect nystagmus $(\mathrm{SDN})$, and neurological nystagmus $(\mathrm{NN}){ }^{1}$ The nystagmus characteristics and waveform of CIN and SDN may not be distinguishable from each other. Typically, the nystagmus is conjugate and purely horizontal in all gaze directions. The intensity may dampen on con- vergence or eye closure, and there may be a region of gaze where it is minimal, or not discernible (null region). The waveform may be pendular, jerk, or a mixture of these, depending on the direction of gaze. If the nystagmus has a vertical component, is horizontal, asymmetric, or unilateral, then a common approach is to investigate for the strong possibility of intracranial pathology. Vertical nystagmus may be pendular, downbeat, or upbeat, or all waveforms may occur in the same subject depending on gaze direction. Vertical pendular nystagmus has characteristics similar to other acquired pendular nystagmus and is often of high frequency and low amplitude. It is associated with a wide range of brainstem and cerebellar disease. Downbeat nystagmus is usually related to abnormalities of the craniocervical junction, but can also occur in cerebellar disease, ${ }^{2}$ with increased intracranial pressure $^{34}$ and secondary to anticonvulsant drugs. ${ }^{5}$ Upbeat nystagmus is a rarer condition and can be associated with cerebellar and pontomedullary abnormalities ${ }^{6-8}$ and as a consequence of drug or organophosphate toxicity. ${ }^{9}$

Asymmetric horizontal or monocular nystagmus often occurs with intrinsic or extrinsic lesions of the optic chiasm, most commonly gliomas or craniopharyngiomas in the paediatric population and thus neuroimaging is necessary in all cases. A condition known as spasmus nutans may also present with asymmetric nystagmus together with head nodding and a head tilt or turn. This is a benign self limiting condition, but the diagnosis is made retrospectively, and on exclusion of neurological and retinal disorders by formal testing. ${ }^{10}{ }^{11}$

We report 14 children who presented to our visual electrophysiology unit at Great Ormond Street Hospital with atypical vertical and asymmetric nystagmus, and who were subsequently diagnosed as having CIN or SDN. Eleven had vertical nystagmus (including two upbeat and one downbeat), and the remaining three had asymmetric horizontal nystagmus. The diagnoses were established by visual electrophysiology testing, clinical examination, and neuroimaging studies. We discuss the important role of both electroretinography (ERG) and visual evoked potentials (VEP) testing in patients presenting with any type of nystagmus.

\section{Methods}

PATIENTS

All patients presenting with nystagmus to the ophthalmology department at Great Ormond Street Hospital for Children are routinely referred for flash ERG, and flash and pattern
Accepted for publication 22 September 1999 


\begin{tabular}{|c|c|c|c|c|c|c|c|c|c|}
\hline Patient & $\operatorname{Sex}$ & Presentation & Onset & Vision last visit & Fundus & Photophobia & $\begin{array}{l}\text { Paradox } \\
\text { pupil }\end{array}$ & Colour vision & Family history \\
\hline 1 & $\mathrm{~F}$ & VN photophobia & 6 weeks & $\begin{array}{l}\text { R 2/36, L } 1 / 24 \\
\text { (Silver Sonksen) }\end{array}$ & normal & yes & no & not tested & none \\
\hline 2 & $\mathrm{~F}$ & VN, lid twitch & birth & $\begin{array}{l}\text { R 4/60, L 3/60 } \\
\text { (Sheridan Gardner) }\end{array}$ & normal & yes & $\begin{array}{l}\text { not } \\
\text { noted }\end{array}$ & identifies red & none \\
\hline 3 & $\mathrm{~F}$ & $\mathrm{VN}$ & 6 weeks & $\begin{array}{l}\mathrm{R} 2 / 18, \mathrm{~L} 2 / 18 \\
\text { (Kay Pictures) }\end{array}$ & normal & yes & yes & not tested & none \\
\hline 4 & M & $\mathrm{VN}$ & birth & $\begin{array}{l}\text { Poor acuity } \\
\text { (Pattern VEPs) }\end{array}$ & normal & yes & $\begin{array}{l}\text { not } \\
\text { noted }\end{array}$ & not tested & $\begin{array}{l}\text { brother BCM (Berson } \\
\text { test +ve) }\end{array}$ \\
\hline 5 & $\mathrm{~F}$ & nystagmus & birth & $\begin{array}{l}\text { R 6/60, L 6/60 } \\
\text { (Snellen) }\end{array}$ & att vessels & yes & $\begin{array}{l}\text { not } \\
\text { noted }\end{array}$ & ishihara $0 / 17$ & none \\
\hline 6 & $\mathrm{~F}$ & $\mathrm{VN}$ poor $\mathrm{F} \& \mathrm{~F}$ & 2 weeks & $\begin{array}{l}\text { moderate/poor } \\
\text { (VEPs 200') }\end{array}$ & normal & yes & no & ishihara $0 / 17$ & brother cone dysf \\
\hline 7 & M & $\mathrm{VN}$ & birth & $\begin{array}{l}\mathrm{R} 3 / 18, \mathrm{~L} 3 / 18 \\
\text { (Snellen) }\end{array}$ & normal & no & no & $\begin{array}{l}\text { ishihara } 0 / 17 \text { Berson } \\
\text { test +ve }\end{array}$ & none \\
\hline 8 & $\mathrm{~F}$ & VN photophobia & $<3$ years & $\begin{array}{l}\mathrm{R} 1 / 18, \mathrm{~L} 1 / 18 \\
\text { (Kay Pictures) }\end{array}$ & $\begin{array}{l}\text { macular reflex } \\
\text { abn }\end{array}$ & yes & no & not tested & none \\
\hline 9 & $\mathrm{~F}$ & Intermittent $\mathrm{VN}$ & 6 months & $\begin{array}{l}\text { R 5/60, L 5/60 } \\
\text { (Snellen) }\end{array}$ & No foveal reflex & no & no & ishihara $0 / 17$ & father squint \\
\hline 10 & M & nystagmus & 5 months & $\begin{array}{l}\text { R 3/60, L 3/60 } \\
\text { (Sheridan Gardner) }\end{array}$ & att vessels & no & $\begin{array}{l}\text { not } \\
\text { noted }\end{array}$ & not tested & none \\
\hline 11 & $M$ & nystagmus & birth & $\begin{array}{l}\text { moderate/poor } \\
\text { (VEPs 200') }\end{array}$ & blonde & yes & $\begin{array}{l}\text { not } \\
\text { noted }\end{array}$ & not tested & none \\
\hline 12 & $M$ & nystagmus & 2 months & $\begin{array}{l}\mathrm{R} 6 / 36, \mathrm{~L} 6 / 36 \\
\text { (Snellen) }\end{array}$ & normal & no & yes & not tested & $\begin{array}{l}\text { male cousin and } \\
\text { grandfather CSNB }\end{array}$ \\
\hline 13 & $\mathrm{~F}$ & $\begin{array}{l}\mathrm{VN} \text {, fixed eccentric } \\
\text { pupil }\end{array}$ & 7 weeks & $\begin{array}{l}\mathrm{R} 6 / 12, \mathrm{~L} 6 / 12 \\
\text { (Snellen) }\end{array}$ & normal & no & no & ishihara 17/17 & none \\
\hline 14 & $\mathrm{~F}$ & nystagmus & birth & $\begin{array}{l}\text { moderate (VEPs } \\
100^{\prime} \text { ) }\end{array}$ & normal & no & no & not tested & none \\
\hline
\end{tabular}

"Vision last visit" If formal visual acuity was not possible to obtain, pattern VEP findings are indicated by stating the smallest pattern size that was resolvable. (Paradox=paradoxical; VN= vertical nystagmus; $\mathrm{F} \& \mathrm{~F}=$ fixing and following; att $=$ attenuated; abn=abnormal; dysf $=$ dysfunction, $+\mathrm{ve}=$ positive; $\mathrm{CSNB}=\mathrm{congenital}$ stationary night blindness.)

VEP testing. Over an 8 year period (January 1991-December 1998) 276 children presenting with nystagmus were recorded in the visual electrophysiology unit. We examined the clinical, electrophysiological, and eye movement details of patients with atypical vertical and asymmetric nystagmus, but who were subsequently diagnosed as having CIN or SDN during this period; 14 patients were identified with such features, and all had undergone several ophthalmological and electrophysiological examinations. There were nine females and five males. The mean age at the first VEP/ERG recording was 20 months. Eleven had formal eye movement tests and nine had undergone neuroimaging studies. The relevant clinical details and history are summarised in Table 1.

VISUAL ELECTROPHYSIOLOGY

VEPs were recorded using silver/silver chloride EEG electrodes placed in a line across the occipital scalp. A midline electrode was sited at $\mathrm{O}_{\mathrm{z}}$ (10-20 system, approximately $3 \mathrm{~cm}$ above the inion) and lateral electrodes were placed midway between the $\mathrm{O}_{z}$ electrode and the ear. All occipital electrodes were referred to a common frontal electrode at $\mathrm{F}_{\mathrm{z}}$ (10-20 system). The flash ERG was recorded from a lower eyelid electrode, also referred to $\mathrm{F}_{\mathrm{z}}{ }^{12}$ Full field pattern reversal VEPs were elicited using a checkerboard pattern presented on a large TV display (subtending 28 degrees horizontal by 21 degrees vertical). Five stimulus check sizes ranging from 25 minutes to 400 minutes of arc were used (25', 50', 100', 200', and 400'). The laboratory was fully darkened and a Grass PS22 photic stimulator lamp, held $15 \mathrm{~cm}$ in front of the eye was used to elicit mixed cone/ rod ERGs. Rod mediated ERGs were elicited using dim blue flash. Cone mediated ERGs were elicited using red flash and bright white flash and $30 \mathrm{~Hz}$ flicker under photopic conditions. ${ }^{13}$

EYE MOVEMENTS

Eye movements were recorded using DC electro-oculography. Silver/silver chloride electrodes were attached with tape to the outer canthi of either eye (to record horizontal eye movements), and infraorbitally and above the eyebrow (to record vertical eye movements) with a reference electrode at the mid-forehead. Patients sat on a parent's lap and the head was held steady by the parent. Video monitoring of the patient was carried out through the entire recording session. ${ }^{14} \mathrm{OKN}$ was elicited by rotating a brightly coloured and patterned, full field curtain around the patient. Leftward and rightward $\mathrm{OKN}$ were recorded at speeds of 25 and $50 \mathrm{deg} / \mathrm{s}$. OKN status was qualitatively assessed as present, or absent/disrupted. ${ }^{15}$

\section{Results}

In all, 276 patients with nystagmus were examined in the visual electrophysiology unit between 1991 and 1998, and 37 (13\%) of these had vertical nystagmus, and $23(8 \%)$ had horizontal asymmetric or monocular nystagmus. We identified that 11 of the patients with vertical nystagmus (30\% of all vertical nystagmus patients) and three of those with asymmetric horizontal nystagmus $(13 \%$ of asymmetric nystagmus) had retinal disease, albinism, or congenital idiopathic nystagmus, based on their ERG/VEP results, ophthalmological findings, and no evidence of neurological pathology demonstrable by neuroimaging or other clinical testing. These 14 patients thus make up $5 \%$ of all nystagmus patients seen in the visual electrophysiology unit. The remain- 


\begin{tabular}{|c|c|c|c|c|c|c|}
\hline Patient & Nystagmus characteristics & Neuroimaging & $E R G$ & $V E P$ & $\begin{array}{l}\text { Horizontal } \\
\text { OKN }\end{array}$ & Diagnosis \\
\hline 1 & $\begin{array}{l}\text { Circumrotatory in primary, vertical in } \\
\text { lateral gaze }\end{array}$ & no & poor cone mediated ERGs & att pattern VEPs to all check sizes & present & $\begin{array}{l}\text { cone } \\
\text { dysfunction }\end{array}$ \\
\hline 2 & $\begin{array}{l}\text { Upbeat in primary and upgaze, } \\
\text { horizontal in lateral gaze }\end{array}$ & MRI: N & poor cone mediated ERGs & att pattern VEPs to all check sizes & absent & $\begin{array}{l}\text { cone } \\
\text { dysfunction }\end{array}$ \\
\hline 3 & Fine vertical pendular & MRI: N & poor cone mediated ERGs & att pattern VEPs to all check sizes & present & $\begin{array}{l}\text { cone } \\
\text { dysfunction }\end{array}$ \\
\hline 4 & Fine vertical pendular & no & poor cone mediated ERGs & att pattern VEPs to all check sizes & not tested & $\begin{array}{l}\text { cone } \\
\text { dysfunction }\end{array}$ \\
\hline 5 & $\begin{array}{l}\text { Asymmetric fine horizontal pendular } \\
\text { mainly in LE }\end{array}$ & $\begin{array}{l}\text { MRI and } \\
\text { CT: } N\end{array}$ & poor cone mediated ERGs & $\begin{array}{l}\text { pattern VEPs to medium and large } \\
\text { checks }\left(50^{\prime}-400^{\prime}\right)\end{array}$ & present & $\begin{array}{l}\text { cone } \\
\text { dysfunction }\end{array}$ \\
\hline 6 & Fine vertical pendular & no & poor cone mediated ERGs & $\begin{array}{l}\text { pattern VEPs to medium and large } \\
\text { checks }\left(50^{\prime}-400^{\prime}\right)\end{array}$ & not tested & $\begin{array}{l}\text { cone } \\
\text { dysfunction }\end{array}$ \\
\hline 7 & $\begin{array}{l}\text { Fine vertical pendular and } \\
\text { circumrotatory, head shake }\end{array}$ & CT: $N$ & poor cone mediated ERGs & $\begin{array}{l}\text { pattern VEPs to medium and large } \\
\text { checks }\left(50^{\prime}-400^{\prime}\right)\end{array}$ & present & $\begin{array}{l}\text { cone } \\
\text { dysfunction }\end{array}$ \\
\hline 8 & Fine vertical pendular & no & poor cone mediated ERGs & att pattern VEPs to all check sizes & not tested & $\begin{array}{l}\text { cone } \\
\text { dysfunction }\end{array}$ \\
\hline 9 & $\begin{array}{l}\text { Vertical pendular, worse in LE, } \\
\text { horizontal in lateral gaze }\end{array}$ & $\begin{array}{l}\text { MRI and } \\
\text { CT: N }\end{array}$ & $\begin{array}{l}\text { poor cone and rod } \\
\text { mediated ERGs }\end{array}$ & $\begin{array}{l}\text { pattern VEPs to large checks only } \\
\left(200-400^{\prime}\right)\end{array}$ & present & $\begin{array}{l}\text { cone-rod } \\
\text { dystrophy }\end{array}$ \\
\hline 10 & $\begin{array}{l}\text { Asymmetric (LE worse) fine horizontal } \\
\text { pendular in primary and L gaze, jerk in } \\
\text { R gaze }\end{array}$ & MRI: N & $\begin{array}{l}\text { poor cone and rod } \\
\text { mediated ERGs }\end{array}$ & att pattern VEPs to all check sizes & present & $\begin{array}{l}\text { cone-rod } \\
\text { dystrophy }\end{array}$ \\
\hline 11 & $\begin{array}{l}\text { Asymmetric fine horizontal pendular } \\
\text { mainly in LE }\end{array}$ & MRI: N & normal & $\begin{array}{l}\text { att pattern VEPs to all check sizes } \\
\text { Crossed asymmetry flash VEP }\end{array}$ & present & albinism \\
\hline 12 & $\begin{array}{l}\text { Vertical upbeat some horizontal } \\
\text { pendular }\end{array}$ & MRI: N & $\begin{array}{l}\text { well preserved a wave, } \\
\text { poor b wave (negative } \\
\text { ERG) }\end{array}$ & $\begin{array}{l}\text { att and delayed pattern VEPs to all } \\
\text { check sizes }\end{array}$ & absent & $\mathrm{X}$ linked CSNB \\
\hline 13 & $\begin{array}{l}\text { Vertical upbeat and pendular, } \\
\text { horizontal jerk on lateral gaze }\end{array}$ & MRI: N & normal & $\begin{array}{l}\text { pattern VEPs smaller than average to } \\
\text { all check sizes }\end{array}$ & present & CIN \\
\hline 14 & Downbeat worse in upgaze & MRI: N & normal & $\begin{array}{l}\text { pattern VEPs smaller than average to } \\
\text { all check sizes }\end{array}$ & present & CIN \\
\hline
\end{tabular}

VEP "attenuated VEPs" refer to clinically significant degradation of the responses, "VEPs smaller than average" implies small responses that are just within the normal amplitude range. $(\mathrm{N}=$ normal; att=attenuated, $\mathrm{CSNB}=$ congenital stationary nightblindness; $\mathrm{CIN}$ congenital idiopathic nystagmus; poor=significantly attenuated or absent.)

ing patients with vertical nystagmus were diagnosed with a range of neurometabolic conditions and intracranial lesions. Of the remaining patients with horizontal asymmetric nystagmus, seven were diagnosed as having "spasmus nutans" and 12 had identifiable intracranial pathology (including chiasmal glioma, craniopharyngioma, and delayed myelination).

Table 2 summarises the diagnoses, electro- physiological, and eye movement findings for all 14 patients.

Patients could be divided into five diagnostic groups:

(1) Eight patients had congenital cone dysfunction (achromatopsia). Seven had vertical nystagmus, and one patient had horizontal asymmetric nystagmus. In all patients, the cone mediated ERGs were not detectable or signifi-
A Control
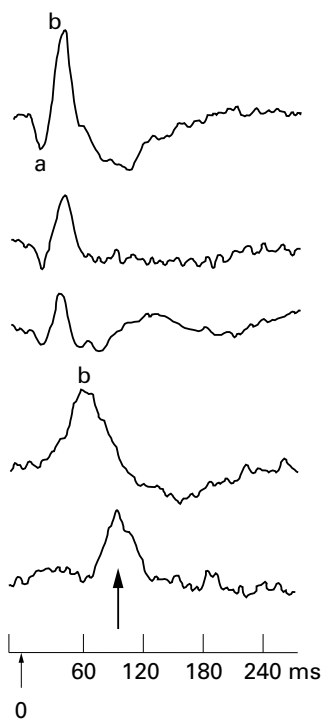

B Cone dysfunction
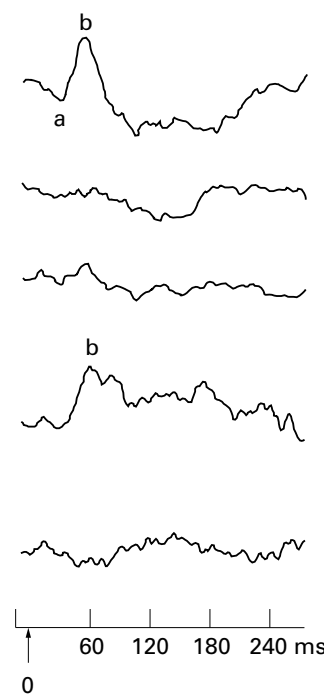

C Cone-rod dystrophy

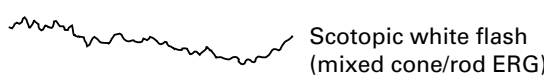

Red flash
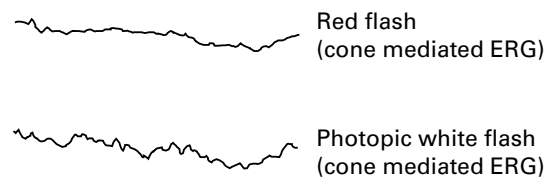

Dim blue flash
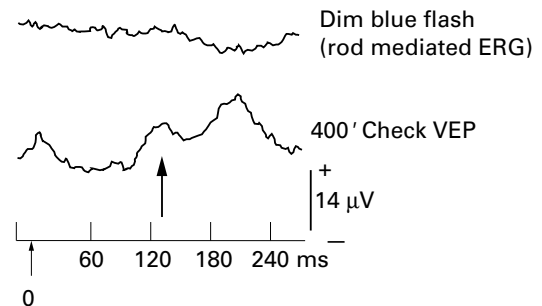

Figure 1 Flash ERGs to scotopic bright white (mixed cone and rod mediated response), red, bright white photopic (both cone mediated responses) and dim blue flash (rod mediated response) from a control subject (A), patient no 2 with cone dysfunction $(B)$ and patient no 10 with cone-rod dystrophy $(C)$. Note the prolonged $b$ wave latency of the scotopic white flash ERG in the patient with cone dysfunction $(B)$ relative to control $(A)$ indicating that it is predominantly rod generated, whereas the cone mediated red and photopic white ERGs are absent. The ERGs under all stimulus conditions are not discernible in the patient with cone-rod dystrophy (C). The bottom traces show the occipital pattern VEPs to 400' checks. The arrows show the major positive component (P100). The cone-rod dystrophy patient (C) shows a degraded and prolonged response (up arrow), reflecting poor macular function, whereas the response is barely discernible for the cone dysfunction patient $(B)$. 
A CSNB patient
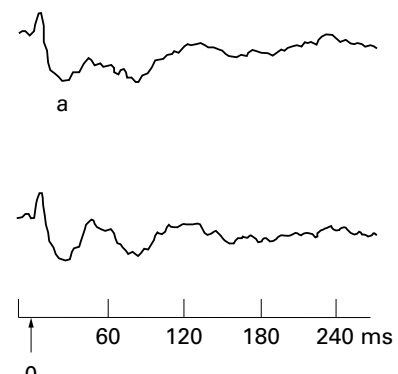

LE ERG

RE ERG

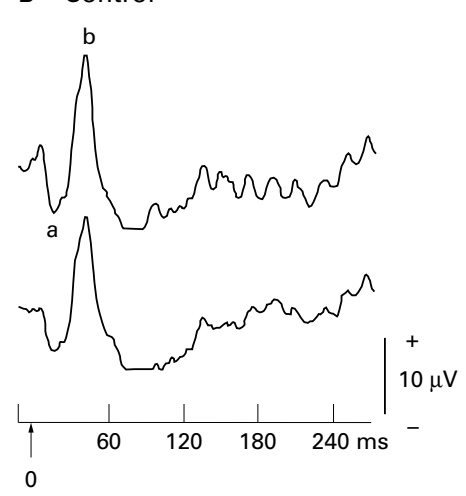

Figure 2 Scotopic bright white flash ERGs from patient no 12 with X linked congenital stationary night blindness (CSNB) (A) and age matched control (B). Note the broadened $a$ wave and absent $b$ wave giving the characteristic "negative" ERG configuration in the patient.

cantly attenuated, whereas the rod mediated responses had a well preserved waveform and were of normal size. Pattern VEPs, particularly to the smaller check sizes $\left(\leqslant 50^{\prime}\right)$, were not usually detectable reflecting poor macular function (Fig 1B). Three patients (patient nos 5, 6, and 7) had partial cone dysfunction as there was evidence of attenuated cone function and pattern VEPs to medium and large sized checks $\left(\geqslant 100^{\prime}\right)$. Two of the patients were male (patient nos 4 and 7) and we tested them for $\mathrm{X}$ linked blue cone monochromatism (BCM) using the Berson colour plates, ${ }^{16}$ as atypical vertical nystagmus has been reported in this rare condition (see Discussion). One patient was too young to complete the Berson test (no 4), but his older brother who is similarly affected (with the same ERG/VEP findings but horizontal pendular nystagmus instead of vertical nystagmus), performed the test well. Both boys were found to have functioning blue cones on Berson colour plate testing and can thus be classified as having X linked BCM. ${ }^{17}$

The total number of patients with congenital cone dysfunction recorded in the visual electrophysiology unit over the period reviewed

A

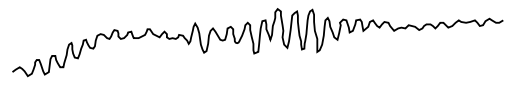

B

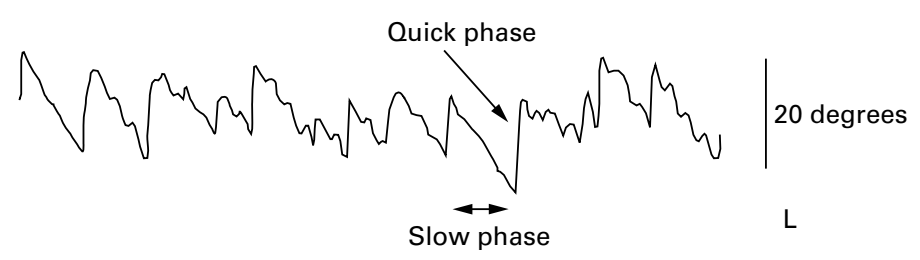

$2 s$

Figure 3 Horizontal eye movement waveforms recorded by bitemporal electro-oculography from patient no 10 with cone-rod dystrophy (aged 7 months) who has high frequency, moderately low amplitude horizontal asymmetric nystagmus $(A)$ and preserved horizontal optokinetic response (B). The optokinetic response to full field leftward curtain rotation can be seen with the pendular nystagmus superimposed upon the slow phases. The ERG and VEP results of this patient are shown in Figure $1 C$. was 98; thus, our data indicate that $8 \%$ of patients with congenital cone dysfunction presented with either vertical or asymmetric nystagmus.

(2) Cone-rod dystrophy ( $n=2)$. One patient had vertical nystagmus, which was also asymmetric (left eye more marked than right eye). The second had fine horizontal pendular nystagmus more marked in the left eye than right. Both cone and rod mediated ERGs were attenuated and the pattern VEPs were markedly degraded in these children (Fig 1C). Their condition appears not to have progressed so far, as the clinical and electrophysiological findings have not altered significantly over a 5 year follow up period.

(3) $X$ linked congenital stationary night blindness $(C S N B)(n=1)$. The patient had large amplitude unusual eye movements which had both a vertical upbeat and horizontal pendular component. The diagnosis was established from the characteristic findings of a negative ERG, with well preserved a wave and very attenuated $b$ wave when tested with bright flash under scotopic conditions (Fig 2). The patient's poor night vision and strong family history of night blindness supported the diagnosis of $\mathrm{X}$ linked CSNB (see Table 1).

(4) Albinism $(n=1)$. The patient had asymmetric fine horizontal pendular nystagmus, predominantly in the left eye. The flash ERGs were normal. The pattern VEPs were very attenuated reflecting poor macular function. Flash occipital VEPs showed the characteristic crossed asymmetry typical of albinism and associated with excessive decussation of monocular fibres at the chiasm. ${ }^{18}$ The electrophysiological findings were confirmed on clinical examination, which showed a blond fundus and mild iris transillumination. Over the period studied, a total of 113 patients with albinism have had electrophysiological testing, but only this one case had an atypical nystagmus.

(5) Idiopathic nystagmus $(n=2)$. One patient had unusual downbeat nystagmus that dampened in downgaze, the other case had a mixture of upbeat and pendular vertical nystagmus in primary and gaze evoked nystagmus in lateral gaze. They were healthy children with normal cone and rod mediated ERGs. The clinical and magnetic resonance imaging findings were all normal, and they were thus diagnosed as having CIN.

Horizontal OKN was tested in 11 patients and was present in nine (82\%) (Fig 3). This finding is unusual as for both CIN and SDN, the OKN tends to be absent. ${ }^{19}$ All three patients with asymmetric horizontal nystagmus had preserved horizontal OKN. Two patients, one with cone dysfunction and the other with CSNB, had absent horizontal OKN and both had a horizontal as well as a vertical component to their nystagmus (see Table 2). Over the 8 year period studied, OKN was tested in 222 patients with CIN or SDN. The vast majority $(n=206,93 \%)$ had absent OKN. Of those with SDN, 23 were diagnosed as having cone dysfunction and nearly half of these cases $(\mathrm{n}=11,48 \%)$ had preserved OKN. 


\section{Discussion}

This report shows that vertical and asymmetrical nystagmus are relatively rare, but a significant proportion (30\%) of congenital vertical and, to a lesser extent (13\%), of asymmetric nystagmus patients have a nonneurological, ophthalmic basis to their anomalous eye movements. Visual electrophysiology is very valuable in helping to diagnose patients with retinal disease and albinism. Normal visual electrophysiology and neuroimaging studies helped establish the diagnosis of CIN in the two patients, who were otherwise healthy children.

Neuroimaging studies will always be essential in patients presenting with vertical and asymmetric nystagmus. However, combined VEP and ERG testing are relatively fast, cheap, and non-invasive tests that are necessary to identify retinal conditions, particularly cone dysfunction and CSNB, and visual pathway anomalies of albinism in young children. In all these conditions the clinical features may not be clear in young children. ${ }^{20}$ If an otherwise well young child presents with congenital atypical nystagmus and has clear electrophysiological changes associated with a retinal disorder or albinism, then neuroimaging studies (which have undesirable risk associated with sedation or anaesthesia, and radiation in the case of CT) would not be indicated.

There have been very few other reports in the literature of vertical familial CIN in patients who had no neurological disease: intermittent upbeat nystagmus was reported in a father and son, ${ }^{21}$ and downbeat nystagmus has been described in an 8 year old boy, whose mother had rotatory and downbeat nystagmus in downgaze. ${ }^{22}$ Vertical pendular nystagmus was described in five members of an Italian family over three generations, ${ }^{23}$ and in two sisters where the pure pendular nystagmus was demonstrated using EOG recording. ${ }^{24}$ An EOG study of congenital upbeat nystagmus in an 11 month old infant with an otherwise normal clinical examination and neuroimaging studies was described by Chaudhry et al. ${ }^{25}$

Unusual eye movements have been reported in two families with $\mathrm{X}$ linked blue cone monochromatism $(\mathrm{BCM}){ }^{26}{ }^{27}$ In this very rare condition, only the blue cones are thought to be functional. Three patients had oblique nystagmus and three obligate female carriers had fine amplitude vertical nystagmus (two upbeat and one downbeat). An earlier study ${ }^{28}$ also reported unusual eye movements in three out of five patients with BCM. Continuous horizontal nystagmus was reported in all BCM and rod monochromatism patients, but the three $\mathrm{BCM}$ also had intermittent, high frequency nystagmus, which was either vertical or torsional. Six of our cone dysfunction patients were female, making the possibility of them having BCM extremely unlikely. However, interestingly, both our male patients with cone dysfunction and vertical nystagmus are most likely to have X linked BCM. One patient correctly identified the Berson plates and the older affected brother of the second case (who was too young to perform the test) also completed the test successfully. ${ }^{17}$

Upbeat $^{29}$ and downbeat nystagmus ${ }^{30}$ have been described as a transient phenomenon in healthy neonates and attributed to immature central vestibular connections associated with vertical canal inputs. Interestingly, there have been two reports describing vertical nystagmus in the neonatal period, which became horizontal by 1 year of age. Hoyt and Gelbart ${ }^{31}$ reported that out of 131 infants with congenital nystagmus, nine presented with a vertical component to their nystagmus: three who presented with an upbeat nystagmus which was replaced by horizontal nystagmus before 1 year of age were diagnosed with Leber's amaurosis. One patient with upbeat had cerebellar vermis hypoplasia, and the remaining five who had seesaw nystagmus were all diagnosed with albinism. Fielder and Evans ${ }^{32}$ also reported two infants, one with upbeat (and normal flash ERGs and VEPs) and the other with pendular vertical nystagmus (and normal flash ERGs but poor VEPs and OKN) which were replaced by horizontal nystagmus by 6 months following the initial onset. These cases differ from our patients in whom the vertical nystagmus persisted well into childhood.

Asymmetric nystagmus was present in three of our patients who were diagnosed with a sensory defect problem. Asymmetric nystagmus in CSNB patients has been reported previously ${ }^{11}{ }^{33}$ but to our knowledge it has not been described in cone dysfunction, albinism or rod-cone dystrophy. Interestingly, all three patients had preserved OKN together with superimposed high frequency low amplitude pendular nystagmus (Fig 3). This oculomotor scenario is similar to that reported in patients diagnosed with spasmus nutans, where the $\mathrm{OKN}$ is preserved and the only abnormality is the presence of the pendular nystagmus. ${ }^{19}$ Moreover, the patients with albinism and rodcone dystrophy also presented with head nodding behaviour and might have been mistaken for spasmus nutans, had the electrophysiology not indicated otherwise.

The optokinetic responses in our group of patients with either vertical or asymmetric, sensory defect or idiopathic nystagmus were atypical and variable. It has been shown that patients with congenital vertical or torsional nystagmus have preserved horizontal $\mathrm{OKN}{ }^{34}$ In our experience, the disruption or absence of the horizontal optokinetic response in subjects with horizontal nystagmus (that is, the $\mathrm{OKN}$ in the meridian of the eye oscillation) is the typical finding in patients with CIN and SDN and is thought to be related to the underlying mechanisms of SDN or CIN. ${ }^{35}$ Out of all the patients with cone dysfunction seen over the 8 year study period, the 11 patients out of 23 who had preserved $\mathrm{OKN}$ are exceptions to this empirical rule. The relatively high percentage $(48 \%, n=11 / 23)$ of preserved horizontal OKN in patients with cone dysfunction, in general, irrespective of the plane of eye oscillation does not support the observation that the optokinetic response is preserved in the orthogonal plane to the congenital nystagmus. Cone 
dysfunction patients often have high frequency, low amplitude pendular nystagmus that may be similar to acquired pendular nystagmus seen in neurological disease. ${ }^{19}$ This is different from the usual CIN/SDN nystagmus waveforms. It remains to be investigated whether the preserved optokinetic response seen in the high proportion of cone dysfunction patients reflects their particular type of nystagmus.

\section{Conclusions}

Patients with congenital vertical and horizontal asymmetric nystagmus are rare, and this type of nystagmus strongly suggests intracranial pathology indicating the necessity for neuroimaging studies. We found that $5 \%(14 / 276)$ of all patients presenting with nystagmus and referred for visual electrophysiological testing had congenital idiopathic nystagmus, albinism, or retinal disease with vertical or asymmetric nystagmus. The majority of the patients in this study (8/14) with vertical or horizontal asymmetric nystagmus had cone dysfunction as revealed by visual electrophysiology testing. Visual electrophysiological tests (flash ERGs and VEPs) are important, non-invasive diagnostic tools for such patients which should ideally be carried out in all children and infants presenting with any type of nystagmus.

We would like to thank the Iris Fund for their support.

1 Casteels I, Harris CM, Shawkat F, et al. Nystagmus in infancy. Br f Ophthalmol 1992;76:434-7.

2 Yee RD. Downbeat nystagmus: characteristics and localization of lesions. Trans Am Ophthalmol Soc 1990; 87:9841032.

3 Phadke JG, Hern JEC, Blaiklock CT. Downbeat nystagmus - a false localising sign due to communicating hydrocephalus. F Neurol Neurosurg Psychiatry 1981;44:459.

4 Lavin PJM, Bahntge MF. Downbeat nystagmus with supratentorial disease. Neuro-ophthalmology 1983;3:235-7.

5 Miller NR. In: Walsh and Hoyt's clinical neuro-ophthalmology. Miller NR. In: Walsh and Hoyt's clinical neuro-oph

6 Kattah JC, Kolsky MP, Guy J, et al. Primary position vertical nystagmus and cerebellar ataxia. Arch Neurol 1983;40 310-14.

7 Baloh RW, Yee RD. Spontaneous vertical nystagmus. Rev Neurol (Paris) 1989;145:527-32.

8 Traccis S, Raced G, Aiello I, et al. Upbeat nystagmus as an early sign of cerebellar astrocytoma. $\mathcal{F}$ Neurol 1989;236 359-60.

9 Jay WM, Marcus RW, Jay MS. Primary position upbeat nystagmus with organophosphate poisoning. F Pediatr Ophthalmol Strabismus 1982;19:318-19.

10 Gottlob I, Zubcov A, Catalano RA, et al. Signs distinguishing spasmus nutans (with and without central nervous system lesions) from infantile nystagmus. Ophthalmology 1990;97:1166-75.
11 Lambert SR, Newman NJ. Retinal disease masquerading as spasmus nutans. Neurology 1993;43:1607-9.

12 Kriss A. Skin ERGs their effectiveness when recording from young children and comparison with ERGs recorded using various types of corneal electrode. Int F Psychophysiol 1994; 16:137-46.

13 Kriss A, Russell-Eggitt I. Electrophysiological assessment of visual pathway function in infants. Eye 1992;6:145-53.

14 Harris CM, Kriss A, Shawkat F, et al. The use of video in assessing and illustrating abnormal eye movements in assessing and illustrating abnormal eye movements
young children. $\mathcal{F}$ Audiovis Media Med 1992;5:113-16.

15 Jacobs M, Harris C, Shawkat F, et al. The objective assessment of abnormal eye movements in infants and young children. Aust NZ F Ophthalmol 1992;20:185-95.

16 Berson E, Sandberg M, Rosner B, et al. Color plates to help identify patients with blue cone monochromatism. Am f Ophthalmol 1983;95:741-7.

17 Haegerstom-Portnoy G, Schneck ME, Verdon WA, et al. Clinical vision characteristics of the congenital achromatopsias. II. Color vision. Optom Vis Sci 1996;73:457-65.

18 Russell-Eggitt I, Kriss A, Taylor D. Albinism in childhood: a flash VEP and ERG study. Br F Ophthalmol 1990;74:136 40.

19 Harris CM. Nystagmus and eye movement disorders. In: Taylor D, ed. Paediatric ophthalmology. Oxford: Blackwell Science, 1997:869-96.

20 Kriss A, Thompson D. Visual electrophysiology. In: Taylor D, ed. Paediatric ophthalmology. Oxford: Blackwell Science, 1997:93-121.

21 Sogg RL, Hoyt WF. Intermittent vertical nystagmus in a father and son. Arch Ophthalmol 1962;68:515-17.

22 Bixenman WW. Congenital hereditary downbeat nystagmus. Can f Ophthalmol 1983;18:344-8.

23 Forsythe WI. Congenital hereditary vertical nystagmus. $\mathcal{f}$ Neurol Neurosurg Psychiatry 1955;18:196-8.

24 Funahashi K, Kuwata T, Yabumoto $M$, et al. Congenital vertical pendular nystagmus in sisters. Ophthalmologica 1988;196:137-42.

25 Chaudhry NA, Gonzalez C, Lee MS. EOG documentation of an unique case of congenital vertical nystagmus. Invest Ophthalmol Vis Sci 1996;37:S278.

26 Gottlob I, Reinecke RD. Eye and head movements in patients with achromatopsia. Graefes Arch Clin Exp Ophthalmol 1994:232:392-401.

27 Gottlob I. Eye movement abnormalities in carriers of bluecone monochromatism. Invest Ophthalmol Vis Sci 1994;35: 3556-60.

28 Yee RD, Farley MK, Bateman JB, et al. Eye movement abnormalities in rod monochromatism and blue-cone monochromatism. Graefes Arch Clin Exp Ophthalmol 1985; 223:55-9.

29 Goldblum TA, Effron LA. Upbeat nystagmus associated with tonic downward deviation in healthy neonates. $\mathcal{F}$ Pediatr Ophthalmol Strabismus 1994;31:334-5.

30 Weissman BM, Dell'Osso LF, DiScenna A. Downbeat nystagmus in an infant. Spontaneous resolution during infancy. Neuro-ophthalmology 1988;8:317-19.

31 Hoyt CS, Gelbart SS. Vertical nystagmus in infants with congenital ocular abnormalities. Ophthalmic Paediatric Genet 1984;6:155-61.

32 Fielder AR, Evans NM. Is the geniculostriate system a prerequisite for nystagmus? Eye 1988;2:628-35.

33 Gottlob I, Wizov SS, Reinecke RD. Quantitative eye movement recordings of retinal disease mimicking spasmus ment recordings of retinal disease mimick

34 Abadi RV, Dickinson CM. The influence of preexisting oscillations on the binocular optokinetic response. Ann Neurol 1985;17:578-86.

35 Harris CM. Problems in modelling congenital nystagmus: towards a new model. In: Findlay JM, Walker R, Kentridge RW, eds. Eye movement research. Mechanisms, processes and applications. Amsterdam: Elsevier Science, 1995: 239-53. 